\title{
Miranda
}

Revue pluridisciplinaire du monde anglophone /

Multidisciplinary peer-reviewed journal on the English-

speaking world

19 | 2019

Rethinking Laughter in Contemporary Anglophone Theatre

\section{Anasyrma et la hantise du rire dans le théâtre de Tennessee Williams}

\section{Emmanuel Vernadakis}

\section{OpenEdition}

\section{Journals}

Édition électronique

URL : http://journals.openedition.org/miranda/20205

DOI : 10.4000/miranda.20205

ISSN : 2108-6559

\section{Éditeur}

Université Toulouse - Jean Jaurès

Édition imprimée

Date de publication : 7 octobre 2019

\section{Référence électronique}

Emmanuel Vernadakis, « Anasyrma et la hantise du rire dans le théâtre de Tennessee Williams », Miranda [En ligne], 19 | 2019, mis en ligne le 09 octobre 2019, consulté le 16 février 2021. URL : http:// journals.openedition.org/miranda/20205 ; DOI : https://doi.org/10.4000/miranda.20205

Ce document a été généré automatiquement le 16 février 2021.

\section{(c) (i) () $\Theta$}

Miranda is licensed under a Creative Commons Attribution-NonCommercial-NoDerivatives 4.0 International License. 


\title{
Anasyrma et la hantise du rire dans le théâtre de Tennessee Williams
}

\author{
Emmanuel Vernadakis
}

Dans A Streetcar Named Desire, Stanley se fâche parce que Blanche, sa belle-sœur, l'a appelé «Polak » : "I am not a Polack. People from Poland are Poles » (scène 8), dit-il. Le verbe « are» - plutôt que «call» (cf. People from Poland are called Poles) - permet ici la condensation de deux sens dans « $\mathrm{P} /$ pole », le deuxième licencieux : «pole is slang for a penis » (Urban Dictionnary). Si le spectateur découvre ce deuxième sens, une autre voie de signification s'ouvre pour permettre à Stanley de se présenter ouvertement à Blanche et aux spectateurs - comme une bête sexuelle. D'un dialogue « réaliste » nous sommes ainsi introduits dans un espace d'échange libre, voire libertin, dont la nature poétique, au lieu de respecter les conventions, les brave. Le temps d'un éclair, l'auteur dévêt un angle voilé de la pièce pour éclairer la nature à la fois " physique » et symbolique d'un personnage qui personnifie la fécondité. On sait - ou on se doute - que Stanley est une bête sexuelle; néanmoins on ne s'attend pas à ce qu'il se définisse de lui-même comme tel. Dans cet exemple, c'est comme si le texte nous défiait de lever le voile de décence que la langue revêt aux situations de communication formelle pour que la «nudité » du personnage concerné se révèle en éclair. Les spectateurs qui lèvent ce défi perçoivent un dénudement; comme si le voile de décence qui revêt le discours de Stanley était soulevé. La déclaration du personnage brise les tabous par synecdoque - la partie pour le tout - pour exposer à nos yeux sa «nudité ». Ce processus mental évoque la gestuelle concrète d'anasyrma, retroussement rituel d'un vêtement dans l'antiquité grecque qui découvrait la nudité du sujet dans un objectif apotropaïque (Vernadakis 2000, 291). L'identité de Stanley se réduit alors à un phallus dévoilé par un anasyrma; ce qui, dans l'espace théâtral, fait écho au lever du rideau et dans l'espace plus large du spectacle, au strip-tease. Cette dimension fortement sexuelle du personnage, révélée par « retroussement »- pensons au French cancan ou à la célèbre image de Maryline dans The Seven Year Itch (1955) - nous réjouit et nous fait rire.

Le rapport au rire ${ }^{1}$ est abordé de manière moins systématique que d'autres aspects de l'œuvre de Williams, comme par exemple l'homosexualité à laquelle il se rattache par 
une sémiotique gothique: invisible, néanmoins puissant, le rire y surgit comme une hantise. La mise en perspective de l'homosexualité par une sémiotique gothique dont les modalités se déclarent sur un plan moins manifeste que symbolique a été étudiée par Brian Peters. Sans aborder le rire, Peters analyse la manière dont la poétique visuelle de l'œuvre engage le langage dans la création d'images textuelles chargées d'érotisme qui recyclent et diffusent le désir² (Peters 4-6). Le rire est suscité précisément par ce type d'images, chargées de sexualité.

3 La sexualité comme ressort du comique, surtout lorsqu'elle se polarise sur le phallus, est un trait caractéristique de l'ancienne comédie attique. Née en Grèce au cinquième siècle avant notre ère, la comédie est issue des $\kappa \tilde{\omega} \mu o l$ (comoi), chants phalliques, joyeux et festifs, qui accompagnent les processions rituelles en l'honneur des dieux chtoniens, essentiellement Dionysos mais pas exclusivement. Elle se développe sur la scène des concours dramatiques autour d'un personnage phallique à travers plaisanteries grivoises ou scatologiques. Les onze comédies d'Aristophane parvenues jusqu'à nous illustrent de manière caractéristique ce trait (Harvey 44, 115-116, 320 ; Hartnoll 406). Leur marque principale est la liberté du langage (Abirached 130) qui donne lieu à des jeux de mots habiles, notamment des jeux d'onomastique, dont, toutefois, l'objectif n'est pas simplement de divertir. Hautement poétique, leur " indécence " est d'une facture artistique qui n'a rien à envier à la tragédie (Mpoukalas, 26). Williams a systématiquement recours à ces pratiques anciennes qui se combinent subtilement à la sémiotique gothique dont parle Peters. Pour étudier le rire invisible de son œuvre, cette sémiotique gothique sera éclairée par une série de références classiques qui nous permettront de déconstruire la condensation poétique extrême de ses œuvres. Le rapport du théâtre de Williams aux origines de la comédie, à l'ancienne comédie attique et aux rituels qui lui sont associés de près ou de loin, comme les chants et processions phalliques des mystères de l'antiquité, constituent un aspect peu étudié de l'œuvre de Williams. Dans l'ouvrage, désormais classique, de Judith J. Thompson, Tennessee Williams' Plays: Memory, Myth and Symbol (Peter Lang 2002), il est question de l'intertextualité mythologique de son théâtre. L'intérêt de Williams pour l'antiquité grecque et sa connaissance de celle-ci n'y sont toutefois pas abordés, le rire non plus. Williams a étudié le grec à Washington University, St Louis et il en parle à plusieurs reprises (Notebooks 67, 87, 89). L'importance qu'il accorde à cette matière est manifeste dans une entrée du 25 janvier 1937 : « Now I must stop and study Greek. Oh, yes, Greek is so important just now. " (Notebooks 73) Sa perception de l'antiquité grecque ne se borne cependant pas à des connaissances universitaires formelles; il s'y intéresse suffisamment pour l'étudier à travers des lectures personnelles ${ }^{3}$. La posture de l'œuvre de Williams face au rire s'inspire de la gestuelle de l'anasyrma, mot grec qui indique le retroussement d'un vêtement plus ou moins long, pour relever ce qui était initialement recouvert. Il trouve sa source dans les processions d'initiation aux mystères éleusiniens où il est associé à Iambé, inventrice de la poésie comique, Iacchos, avatar de Dionysos, dieu lumineux et tapageur du rire et Baubô, vieille nourrice qui révèle sa nudité à une déesse pour la faire rire (Vernadakis 2000, 291-293 ; 2032-33). L'intérêt de Williams pour ce geste indique un aspect cérébral ou érudit, parfois contestét, de son théâtre. Selon mon hypothèse, le rire chez Williams émerge d'un anasyrma, geste initialement féminin effectué devant une assemblée d'hommes (Vernadakis 2000, 294). Connu pour avoir repoussé des héros et des fléaux naturels, l'anasyrma a une propriété apotropaïque ${ }^{5}$. Cependant, ainsi que le stipule l'épisode d'Iambé, alias Baubô, rapporté par Clément d'Alexandrie, lorsqu'il est exécuté devant une autre femme, l'anasyrma 
déclenche le rire (Vernadakis 2000, 292). Clément rapporte l'histoire de Baubô, une vieille nourrice d'Eleusis qui accueille Déméter en deuil de sa fille et lui offre le kykéon, breuvage régénérateur que la déesse éplorée dédaigne de prendre. Baubô retrousse alors ses vêtements et expose sa nudité : de son sexe surgit Iacchos, fils de la déesse et avatar de Dionysos, qui rit et gigote. Déméter rit, à son tour, et accepte le kykéon. L'anasyrma qui, dans ce mythe, sauve l'humanité de la famine par le rire, déclenche aussi un rire salutaire dans le théâtre de Williams. L'esprit de Baubô l'éleusinienne sera débusqué dans Bobo, l'oiseau jaune qui préside la destinée d'Alma Tutwiler dans la nouvelle « The Yellow Bird », et celui d'Iacchos, avatar de Dionysos et dieu lumineux du rire, dans Oliver Winemiller, protagoniste de "One Arm». Les deux figures mythologiques œuvrent de pair dans Summer and Smoke dont l'héroïne, Alma Winemiller, réunit dans son nom ceux des protagonistes des deux nouvelles précédentes. Verbal, plutôt que gestuel, l'anasyrma déclenche un rire qui émane de la théâtralisation d'un «je " d'artiste. C'est un rire chthonien qui ne se révèle pas d'emblée parce qu'il procède des agissements d'un dramaturge filou qui cherche à " rouler » la censure autant que le désir de divertissement du public. Si la vigilance de l'une et les attentes de l'autre sont satisfaites, elles le sont par tromperie. La cohésion obtenue par l'expressionnisme qui revêt le réalisme et le naturalisme scénographiques des pièces est un masque conçu pour plaire au public et leurrer la censure. Motivées par le rire, les œuvres que nous étudierons procèdent à ces travestissements afin de refondre en drames sociaux les conflits internes de l'écrivain en herbe. Dans ce contexte métafictionnel d'écriture de soi, le rire est celui du dramaturge victorieux, triomphant des forces obscures du puritanisme, forces néanmoins domestiques qui ont nourri son âme. Si bien que ce rire d'auteur triomphant résulte d'un acte violent qui repose sur la capitulation et l'exécution de quelque chose de cher chez le jeune homme - pour utiliser une formule de Wilde, Williams rit en tuant ce qu'il aime ${ }^{6}$. Le souvenir de Wilde, de l'individu et de son œuvre, participe largement au processus. Les pièces de Williams traversent au moins deux filtres, très hétéroclites, pour parvenir à générer le rire : l'œuvre d'Oscar Wilde et les comics'. On s'attachera surtout au premier et surtout à la danse que Wilde a imaginée pour sa pièce française, Salomé (1893), «la danse des sept voiles", striptease symbolique inspiré par la descente aux enfers d'Ishtar (Vernadakis 2003, LXV). Il s'agit d'un motif finement masqué et récurrent chez Williams qui fait le lien avec l'anasyrma de Baubô ${ }^{8}$. Avant de commencer l'analyse des trois œuvres, il convient de vérifier rapidement ce point.

4 Les protagonistes de «The Yellow Bird» et de Summer and Smoke partagent le prénom Alma, dont la sonorité évoque vaguement «Salomé ». En tant que nom commun, en anglais, alma signifie danseuse orientale (Webster's 1913). Dans la nouvelle, le rapport d'Alma à la danse est visuellement évoqué par le verbe "juking ", néologisme dérivé des Juke-boxes devant lesquels Alma se met systématiquement à danser : "She picked up men on the high-way and went out "juking" with them " (Williams 1996, 252). Ce rapport à la danse est aussi mis en scène dans Summer and Smoke où Alma a un double antithétique, un doppelgänger dans le personnage de Rosa, danseuse de flamenco qui se donne en spectacle au Moon Lake Casino, propriété de son père. À travers sa transformation de fille de pasteur en fille de joie, Alma adopte progressivement les traits de Rosa la danseuse. Si bien qu'à la fin de la pièce, elle invite un étranger au Moon Lake Casino, on présume pour «danser» (Williams 2000, 642). Pour ainsi dire, il y a donc un peu de la danseuse Salomé dans Alma. 
5 Pour reprendre, le rire chez Williams se comporte comme un clandestin. Il se cache pour se déclencher une fois que le pathétique se métamorphose en matière métafictionnelle. Infernal et ingénu, primitif et cérébral, il agit tel un spectre espiègle mais salutaire, sans se montrer ; tout comme l'oiseau jaune de la nouvelle « The Yellow Bird».

«The Yellow Bird» est une farce narrativisée. Elle s'articule autour de Bobo, oiseau jaune qui tient les fils de l'intrigue, même si sa propre existence tient, elle aussi, à un fil. Si ses actions ponctuent le récit, son existence est problématique. Bobo n'est littéralement présent qu'à travers son nom qui apparaît uniquement au début et à la fin de la nouvelle. Dans le premier paragraphe, le récit nous plonge dans les origines hawthorniennes de la littérature américaine par la relation qu'il tisse entre Bobo et le passé de la famille de l'héroïne, Alma Tutwiler : la première ancêtre américaine d'Alma aurait été pendue aux procès de sorcellerie de Salem, accusée de s'entretenir avec Bobo, oiseau jaune messager du diable. Dans le dernier paragraphe, Bobo se voit projeté vers l'éternité : son nom est gravé sur le marbre d'un monument élevé en l'honneur d'Alma - un dauphin chevauché par trois personnages androgynes dont le premier porte une croix, le deuxième une lyre grecque et le troisième une corne d'abondance. Entre le début et la fin du récit il n'est pas question de Bobo ; mais on se doute que les transformations d'Alma - fille de pasteur, prostituée, mère, sainte, monument, légende - se font sous l'égide de Bobo.

7 La navigation entre la " préhistoire » américaine - le Salem hawthornien - et l'éternité - symbolisée par le monument funéraire - pourvoient Bobo d'une qualité oxymorique : elle couvre le temps « historique » et son prolongement imaginaire étant partout sans se montrer. Son omniprésence absente témoigne du remarquable degré de condensation poétique atteint par le texte. Car, en effet, lorsqu'on considère les étendues couvertes par le récit sur le plan de la représentation des lieux, du temps et des personnages ${ }^{9}$, les huit pages de la nouvelle enveloppent une substance qui conviendrait mieux à une saga. Si le rythme rapide de l'action, l'aspect grotesque des personnages et leurs nombreuses entrées et sorties (Williams 2012, 251) relèvent de la farce, l'intrigue rattache la nouvelle non seulement au théatre mais aussi à d'autres genres, comme la biographie, et à d'autres modes, comme le fantastique. Ainsi, marquée d'un "réalisme» conventionnel, la trame biographique du récit saute subitement dans le fantastique quelques paragraphes avant la fin: "Now from this point on the story takes a strange turn that may be highly disagreeable to some readers, if any still hoped it was going to avoid the fantastic. » (Williams 2012, 254) De plus, les évènements de l'intrigue qui se déroulent dans le Sud des États Unis, à la Nouvelle-Orléans, puis dans le Nord où Alma devient riche, cartographient l'itinéraire biographique de Williams dans un ordre chronologique ${ }^{10}$. On peut donc soupçonner qu'il y a également des éléments d'écriture de soi dans la biographie d'Alma - d'autant plus que celle-ci sert de modèle à cette autre Alma, protagoniste de Summer and Smoke, toujours fille de pasteur qui se transforme, elle aussi, en prostituée, et au sujet de laquelle Williams dit: "Alma of Summer and Smoke is my favourite-because I came out so late and so did Alma, [...] Miss Alma grew up in the shadow of the rectory. And so did I. » (Devlin 228) Le lecteur des carnets de Williams est en mesure de remarquer nombre d'autres affinités entre le personnage et son auteur ${ }^{11}$. Si Williams s'identifie à Alma, comme il le déclare ici, au lieu de suivre un seul fil rouge dans sa lecture de « The Yellow Bird", il est possible d'en suivre deux: le premier nous guide à travers la 
trajectoire d'Alma, fille de pasteur qui se transformer en fille de joie; le second à travers la trajectoire de Tom Williams qui se métamorphose en Tennessee Williams, destinée toute tracée qui va de l'apprentissage du métier à la consécration du dramaturge (Vernadakis 2013, 49-61).

Par un processus de condensation, propre surtout à la nouvelle, Williams a recours à une stratégie de représentation qui investit deux niveaux d'action; le premier littéral, extérieur et social, le second implicite, intérieur, voire hermétique, et autobiographique. Cela est d'abord travaillé dans les nouvelles, puis dans ses pièces.

Comme plusieurs dramaturges, Goethe, Gogol, Pouchkine, Tchekhov, Wilde, Brecht, Pirandello, Beckett, Pinter, Miller etc., Williams cultive, lui aussi, la nouvelle. Cela se justifie parce que la fiction brève - surtout la nouvelle - et le théâtre se rencontrent sur quelques points qui émanent de contraintes formelles similaires. Par exemple, depuis la naissance du théâtre, la durée de la représentation d'une pièce ne dépasse généralement pas les deux ou trois heures ${ }^{12}$. Ce qui correspond plus ou moins au temps de lecture d'une nouvelle qui, comme l'a souligné Poe, se lit d'un seul trait (May 60-61). De même, associé au temps limité de la représentation, l'espace réduit de la scène incite le dramaturge à recourir à des artifices comparables à ceux du nouvelliste qui ne doit pas dépasser un certain nombre de signes prédéfinis pour respecter les ordres d'un éditeur, les besoins d'une revue, les règles du genre. Cette nécessité de concentration de contenu fait que la création du dramaturge et celle du nouvelliste reposent sur un défi poétique partagé : signifier plus en exposant moins.

Chez Williams, la condensation poétique se produit d'abord dans les nouvelles pour être, seulement ensuite, reprise dans les pièces. Elle met en œuvre un paradigme autobiographique sous forme de dévoilements instantanés indépendants, déclenchés à partir de montages poétiques à sens double ou multiple. Rattachés entre eux selon une logique indépendante de l'intrigue, ils évoluent en parallèle sur une trame implicite qui forme un deuxième récit. Invisible sur le plan littéral, ce récit hante le texte de propos, d'images et d'autres constructions figurées ou intersémiotiques dont la signification est parfois contraire à ce qui est littéralement signifié. La logique de progression discursive séquentielle de ces dévoilements, repérables à tout moment, se présente ainsi comme un double du récit. C'est un double démystifiant et comique. Par exemple, c'est par une série de condensations poétiques sur cette deuxième voie que l'héroïne de « The Yellow Bird » explicite la signification de son prénom, Alma, en anglais ${ }^{13}$, en latin, en grec, en hébreu et en espagnol, au moyen de représentations comiques narrativisées (Vernadakis 2013, 49-61). Ce sont tout d'abord l'onomastique et l'hétéroglossie qui éveillent les qualités performatives du discours narratif pour nous faire découvrir les mises en scène des différents sens du prénom Alma au cours des métamorphoses du personnage. Sur le plan du récit manifeste, Alma se met à fumer à outrance - voire à se droguer si l'on tient compte du double sens du mot Coke: "They had seen her smoking in the White Star drugstore while she was having her afternoon Coke » (Williams 2012, 250). La symbolique de la fumée, reprise pour la refonte dramatique de "The Yellow Bird » dans le titre Summer and Smoke, signale la métamorphose de la matière consumée d'Alma - sa foi puritaine - en un élément nouveau - en énergie vitale. En effet, le texte stipule que le renoncement à son éducation puritaine de vieille fille donne lieu à une énergie sans pareille chez une Alma « flambeuse » qui, grâce à la foi vigoureuse de ses ancêtres, fait sensation : "It was impossible to see how one human constitution could stand up under the strain of so much running around to night places, but Alma had all 
the vigor that comes from generations of firm believers. It could have gone into anything and made a sensation. Well, that's how it was. There was no stopping her once she got started "(Williams 2012, 252). Avec ces propos le narrateur dévoile une exploitation rabelaisienne de la formule latine (d'où l'hétéroglossie) Alma mater, « mère nourricière » : les « cendres » d'Alma-la-puritaine nourrissent l'énergie sexuelle d'Alma la fille-de-joie. Cette performance narrativisée relève de l'autobiographie. Pour peu que l'on connaisse la vie de Williams, on reconnaît des résonances entre celle-ci et l'épisode précédent et percevons également le processus de condensation poétique à l'œuvre dans la nouvelle : élevé à l'ombre du presbytère, comme il le formule dans la citation précédemment utilisée, Williams s'est, comme Alma, adonné à la cigarette et à la drogue (Lahr 236, 280, 285, Smith-Howard 12). Du latin à l'hébreu, alma est aussi un mot de l'Ancien Testament qui intègre la tradition occidentale par l'intermédiaire du grec $\pi \alpha \rho \theta \varepsilon ́ v o \varsigma, ~ " v i e r g e$ ", ainsi qu'alma a été traduit dans le verset d'Isaïe VII, 14-15 sur lequel repose la doctrine de la virginité de Marie. Il se trouve que, sur la deuxième trame narrative, la vie d'Alma parodie au moins deux épisodes de la vie de la Vierge Marie, l'Immaculée Conception et la Dormition. Le puritanisme d'Alma confère de la pureté au physique du personnage qui, malgré le temps qui passe et sa vie de débauche, respire la pureté et l'innocence d'une vierge. Son miroir renvoie à Alma l'image d'un visage virginal qui n'accuse ni les effets du temps ni les excès physiques : « It might have seemed to some people that Alma was living a wasteful and profligate existence, but if the penalty for it was death, well, she was a long time dying [...] It apparently did not have a dissipating effect on her [...] it wouldn't [...] Her face had a bright and innocent look in the mornings » (Williams 2012, 239). En évoquant le célèbre héros débauché d'Oscar Wilde, Dorian Gray, dont le physique reste inaltérable malgré le passage du temps, ce propos inaugure une mise en scène parodique d'Alma en tant que Vierge ${ }^{14}$. Mais il s'agit aussi d'une mise en scène de Tom tel qu'il se décrit en octobre 1941 , lorsqu'il est en train de rédiger la nouvelle : «I look in the mirror. My face is fresh and glowing. I look young again. And pretty! » (Notebooks 245). La chambre d'Alma à la Nouvelle Orléans est décrite comme une "crèche »: «[a] miserable little furnished room-or crib, as it actually was " (Williams 2012, 238). Alma y reçoit une émissaire de ses parents qui constate que dans ce lieu tout est sale et maculé : "The bed was unmade and looked as if it had been that way for weeks. The two-burner stove was loaded with unwashed pots in some of which grew a pale fungus [...] the doors of the big armoire hung open on white summer dresses that were covered with grass stains. $\gg^{15}$ (Williams 2012, 237-238). Parodie de l'Immaculée Conception, cette visite se parachève avec l'annonce de la "bonne" nouvelle - le sens du mot " évangile » en grec - : "Shortly thereafter Alma discovered that she was becoming a mother " (Williams 2012, 238). Comblée de richesses grâce à son fils, enfant ensorcelé qui, nourrisson, sort de la maison en rampant pour aller à la mer et en revenir les mains pleines de trésors marins, Alma s'en va vivre " up North". La résonance ici entre le récit fantastique et la vie de Williams est que, du Sud, l'auteur a déménagé à New York lorsque ses pièces lui ont valu la reconnaissance du public et la prospérité. Une parodie du récit de la Dormition et ascension de Marie clôt cette partie, inspirée par les apocryphes où la Vierge est miraculeusement rapprochée de Jean (Rhodes 194-277). Tandis qu'elle agonise, l'héroïne souhaite que son fils, John, parti dans la marine, vienne à son chevet; mais, au lieu de cela, son lit la conduit à John, son amant préféré qui, tel Neptune, l'accueille au milieu de l'océan, tenant une corne d'abondance remplie de joyaux récupérés dans les épaves de galions espagnols. Il couvre le corps d'Alma de ces joyaux et, au lieu de l'envoyer au ciel, l'emmène avec lui 
dans les profondeurs marines (Williams 2012, 239). Loufoques et fantasmatiques, ces mises en scène du prénom d'Alma qui font d'elle un avatar de Marie, corroborent le lien autobiographique complexe qui rattache le récit manifeste aux dévoilements du récit latent et Alma, la prostituée, à Williams, l'auteur «croustillant » : la métalepse, figure de rhétorique qui dévoile l'intrusion de l'auteur - et pas du narrateur - dans sa fiction (Genette 31 passim), trouve une figure particulièrement convenable dans Marie. La Vierge, qui sert de modèle à la fabrication de la sainte courtisane de Williams est par définition, une figure métaleptique : en tant que mère de Dieu, elle est créatrice de son créateur. Il en est de même pour Alma qui, dans cette nouvelle, fonctionne comme une figure matricielle de son auteur (Vernadakis 213, 52). Ses "performances" de courtisane engagent un texte narratif dans la création d'une identité théâtrale, celle d'Alma, certes, mais aussi celle de son auteur dont la « personne » est créée par le récit de son personnage. La révélation de cette inversion où l'auteur figure comme la construction de sa création, suscite le rire sinon du lecteur du moins de l'auteur qui ruse ostensiblement aux dépens du premier tout en se positionnant contre ses attentes : «Now from this point on the story takes a strange turn that may be highly disagreable to some readers. » (238) Pour aller vite, sur la trame implicite de la nouvelle, cette inversion est un coming out inattendu imposé au lecteur. La surprise contrariée des lecteurs à cette révélation produit le rire de satisfaction de l'auteur triomphant, un rire dont le dauphin "arrogant » (239) du monument funéraire élevé à la mémoire d'Alma fait peut-être écho. Depuis l'antiquité, mais aussi selon les constats récents de certains chercheurs, le dauphin est perçu comme l'un des rares animaux qui non seulement ont une figure rieuse mais sont aussi capables de rire ${ }^{16}$. La manière dont ses contemporains décrivent le rire de Williams n'est pas sans évoquer le « rire » des dauphins ${ }^{17}$ tel qu'on le trouve filmé dans nombre de clips en ligne: "what Mr. Williams's colleagues remember best was his marvelous laugh - a nervous, funny laugh, half-way between a guffaw and a cackle, that erupted at the most improbable times » (Kakutani).

Nous pouvons faire le même type de constats à partir de la nouvelle "One Arm ". Boxeur de la marine qui a perdu son bras dans un accident de voiture, Oliver Winemiller, le protagoniste, se prostitue, comme Alma, mais pour une clientèle homosexuelle. La polysémie du titre ( $\mathrm{arm}=\mathrm{membre} / \mathrm{arme})$ ouvre un horizon d'attente à double entrée où le handicap-le bras amputé-rivalise avec la violence signalée par l'instrument de combat. Une deuxième intrigue qui évolue de manière latente, est encore éclairée par l'onomastique et l'hétéroglossie. Comme dans "The Yellow Bird", l'hétéroglossie double le sens d'un nom propre - ici d'un toponyme - dont la signification en langue étrangère dévoile des " performances » invisibles sur le plan du récit manifeste. Ainsi, une nuit, après avoir tué un de ses clients sur un yacht, oliver Winemiller quitte le bateau en plongeant dans les eaux de Palm Beach (Williams 2012, 198-199). Ce plongeon entame, pour ainsi dire, une «descente aux enfers» pour Oliver qui se traduit concrètement par une série d'étapes qui vont de mal en pis: arrestation, procès, condamnation et exécution suivie de la dissection de son cadavre. Le toponyme Palm Beach ouvre à la fois sur le symbolisme issu de sa première composante, palm, et sur la descente aux enfers de Dionysos suggérée par la contextualisation de l'action. Pour commencer, palm le palmier, terme récurrent chez Williams, est systématiquement utilisé dans sa fiction par le truchement de l'hétéroglossie, à travers le sens de sa traduction grecque : "phénix ». En grec, ce mot désigne à la fois le palmier et l'oiseau mythologique égyptien qui renaît de ses cendres. Le deuxième sens de ce mot (i.e. phénix) fait que le plongeon d'oliver dans ces eaux 
préconise le contraire de ce qui est exposé par l'action. La descente aux enfers du personnage est en quelque sorte devancée par l'annonce d'une régénération qui va à rebrousse-poil de l'action et qui repose sur un hypotexte comique scabreux. Dieu du vin, Dionysos habite le nom Wine-miller qu'oliver partage avec l'Alma de Summer and Smoke. La descente aux enfers de Dionysos était célébrée dans l'antiquité au cours des mystères de Lerne, par une cérémonie d'évocation nocturne, le rite des Nyctelia, au lac Alcyonien, réputé pour communiquer avec le royaume d'Hadès ${ }^{18}$. Selon la légende à l'origine de ce rituel, pour descendre aux enfers, Dionysos demande l'aide de Prosymnos, un vieux berger de Lerne qui connaît bien le lac Alcyonien. En récompense pour son aide, le jeune dieu promet de se prêter aux désirs de Prosymnos, une fois rentré. Cependant il ne peut tenir sa promesse car Prosymnos meurt avant. Pour tenir malgré tout son engagement, Dionysos taille un morceau de figuier en forme de phallus et, assis sur la tombe de Prosymnos, s'acquitte de sa dette (Clément d'Alexandrie, Discours aux Gentils 34, Grimal 127, Callimach, 125). La résonance de cet épisode dans la nouvelle se manifeste par la volonté d'oliver d'honorer sa dette morale à l'égard de ses anciens clients, à l'origine méprisés parce qu'homosexuels. Le phallus en bois est peutêtre reflété par une punaise inversée, dessinée au milieu de la chaise électrique dans un croquis du type comics, élaboré par Oliver pour l'envoyer en réponse aux lettres que ses anciens clients lui écrivent en prison.

Annoncée par le phénix « caché » dans Palm Beach, la régénération du personnage se produit au moyen de ces lettres de soutien. Elles permettent à Oliver de comprendre qu'il a, malgré tout, compté dans la vie de ses correspondants (Williams 2012, 200). Elle s'opère aussi, de manière plus radicale et comique, à la fin de la nouvelle par les implications métatextuelles du personnage central qui, comme il a été suggéré par ailleurs, s'offre comme une personnification ou une métonymie de l'œuvre de Williams (Vernadakis 2016, 144-145). Ces implications sont introduites notamment par un télescopage de l'œuvre littéraire de Williams et de l'œuvre graphique d'oliver, à travers "the hot seat", ce croquis de type cartoons dont il a été question plus haut. Cela représente une chaise électrique. Ambigu, son titre associe mort, sexualité et volonté de puissance. Inspiré de la Chaise de l'artiste (1888-9) de Van $\mathrm{Gogh}^{19}$, ci-contre à droite,

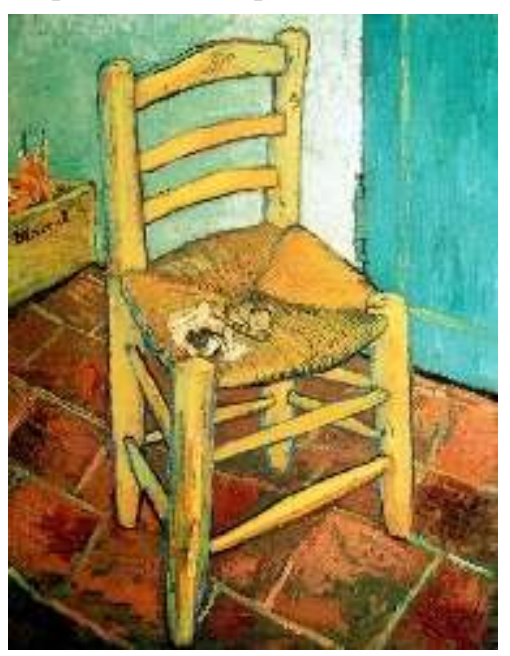

Van Gogh. La chaise de l'artiste, https://www.wikiart.org/en/vincent-van-gogh/van-gogh-s-chair-1889, domaine public.

ce croquis superpose une œuvre dont Williams est (forcément) l'auteur effectif - celle qui est reproduite dans le volume (Williams 2012, 202) et ci-contre, à gauche, 


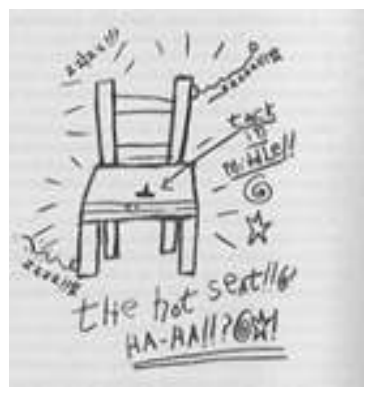

T. Williams. The Hot Seat.

14 à celle dont Oliver est l'artiste présumé - celle envoyée aux correspondants. Si la chaise est censée représenter l'instrument d'exécution d'oliver, le croquis reprend sur le plan de l'image la stratégie de condensation sémantique par ambivalence que l'on a constatée sur le plan textuel. Ainsi que l'annonce le titre «the hot seat", cette chaise peut être vue de plusieurs manières: instrument d'exécution, certes; toutefois, les rayons qui l'entourent comme un halo, les spirales et étoiles qui «évoluent» autour d'elle l'associent également à la lumière des astres, voire à un astre (cf. l'expression une "star »), en reprenant ainsi ce que le texte relate de la manière dont Oliver était vu par ses anciens clients " [the youth] stood like a planet among the moons of their longing " (Williams 2012, 202). Sur la partie basse du croquis figure également un "HA-HA! » imposant, souligné et suivi de spirales et d'étoiles telles que l'on en trouve dans les bandes dessinées pour exprimer ce qui ne peut pas s'écrire, la colère ou des mots licencieux. Par les rayons de lumière, les étoiles et le rire qui l'entourent, la chaise peut être aussi associée à la symbolique d'Iacchos, le personnage qui, on l'a vu, sort du sein de Baubô comme un prolongement phallique de son corps, pour faire rire Déméter. Onomatopée du rire - en grec kaүxáł $\omega$ - Iacchos, le porteur de lumière des mystères d'Eleusis (Grimal, 220), aussi appelé " l'astre lumineux » des mystères d'Eleusis (Aristophane 171-172), est un avatar de Dionysos. Dans Lady Chatterley's Lover de D. H. Lawrence, auteur particulièrement apprécié par Williams (Débusscher 167), Connie Chatterley, dont l'amant s'appelle Oliver Mellors, fait d'Iacchos une personnification du phallus ${ }^{20}$ (Lawrence 219, Vernadakis 2016, 148). Les échos entre Oliver Mellors et Oliver Winemiller ne sont pas seulement phonétiques : les deux Oliver ont eu une liaison avec la femme du propriétaire qui les embauchait. Ce qui pourrait signifier que Williams aurait initialement découvert la figure d'Iacchos chez Lawrence. De manière osée, néanmoins comique, la punaise inversée au milieu de la chaise remplace chez Williams la pipe de Van Gogh, pour faire d'abord le lien entre art et plaisir sexuel, puis entre violence et volonté de puissance. Un symbole phallique et par procuration le plaisir qui s'y rattache siègent ainsi dans le croquis de Williams; ce qui pourvoit le «hot » du syntagme the hot seat, d'un troisième sens, cette fois sexuel. Par le biais de la langue, de manière métonymique le siège désigne le pouvoir de l'individu qui l'occupe ("seat», représenté de manière plastique en tant que " chair » = a person in charge of...). La chaise d'Oliver, qui éclaire l'espace de son halo, peut être aussi le siège de l'artiste Williams, sa chaire, et Oliver, condamné à trôner sur celle-ci, une représentation métonymique de l'œuvre des deux. Comme Oliver, la chaise associe le pouvoir idéologique du mythe à la question d'identités sexuelles et à la littérature du Sud dont la fragilité, représentée par le corps diminué d'Oliver, constitue aussi le pouvoir. Cette fragilité magique car puissante - qui se retrouve par ailleurs chez Laura de The Glass Menagerie $e^{21}$ - incarne l'homosexualité de Williams, perçue comme une "volonté de puissance " créatrice ${ }^{22}$ 
déterminante pour l'œuvre (Vernadakis 2016, 152). La vision d'une nature homosexuelle fragile face à une virilité puissante est stéréotypée mais nécessaire pour l'articulation des conflits dramatiques de l'œuvre, sur le plan de leur littéralité, parce qu'elle répond mieux aux attentes du public. Les pièces de Williams redressent le stéréotype, par le fait que tous les personnages sont, dans une certaine mesure, des parcelles de la personne de l'auteur qui se représente aussi bien à travers leur violence qu'à travers leur fragilité. Ce qui constitue une nouvelle source de rire - du moins pour Williams lui-même.

L'aspect pathétique de la nouvelle, qui se termine avec le cadavre d'oliver en attente d'être disséqué, s'avère du coup comique : car si le corps d'oliver est aussi le corpus de Williams, les étudiants de la faculté de médecine qui n'osent pas le disséquer pour l'étudier, tant il est beau, sont moins réticents que nous, vous et moi, qui sommes en train d'éplucher, ici même, à travers la lecture de ces pages, une portion de ce corpus pour l'observer sous une loupe scientifique. Williams nous a « roulés ». Nous pouvons, à notre tour, imaginer le rire de l'auteur devant ce spectacle figuré par anticipation nous en train de disséquer son œuvre. Ce serait un rire d'outre-tombe qui résonnerait un peu, imaginons-le, comme celui de Muttley < https://gfycat.com/fr/gifs/detail/ ignorantrespectfulerin>.

Un dernier exemple de "The Yellow Bird", nous permettra d'illustrer la manière organique dont les deux intrigues parallèles, manifeste et latente, fonctionnent dans la logique de condensation poétique précédemment esquissée pour que le texte soit ouvertement exposé à la contradiction sans qu'il y ait incohérence. Sur le plan thématique, l'intrigue manifeste s'engage dans une critique sociale; l'intrigue latente dans une écriture de soi - qui dévoile le rire.

La subite transformation d'Alma en prostituée se déclenche par un dimanche estival où la chaleur est insupportable. Pour arrêter son mari, le pasteur, dans un trop long sermon, la mère d'Alma agite si violemment la feuille de palmier qui lui sert d'éventail que celle-ci finit par tomber en lambeaux : « The minister's wife had plucked nervously at the strings of her palm-leaf fan till it began to fall into pieces " (Williams, 2012, 249). Le recours à l'hétéroglossie qui, comme déjà constaté, fait aussi du palmier un phénix se précise avec la phrase qui suit: "...the interior of the church was yellow oak, a material that made you feel as if you were sitting in the middle of a fried egg " (Williams 2012, 249). L'œuf au plat en question est, sans doute, celui de l'oiseau jaune du titre : il ne s'agit pas d'un bobolink, en français goglu des prés, mais d'un phénix. Cette comparaison de l'église à un œuf de phénix a une portée métaphorique qui ouvre sur la trame autobiographique et invisible, pour indiquer la naissance " $a b$ ovo » d'une nouvelle Alma, sous l'égide de Bobo - ou de Baubô (?). Les événements qui évoluent sur le plan de l'intrigue latente ne sont pas relatés par le narrateur; ils sont plutôt construits par le texte. Ils « doublent » ceux de la narration en évoluant à contre-sens de la volonté des personnages secondaires et de l'intrigue manifeste pour montrer que rien ne peut arrêter la trajectoire d'Alma dans son processus de devenir prostituée, puis sainte, ou Tom dans son processus de devenir dramaturge, puis d'être consacré en tant que tel. Par exemple, lorsqu'Alma se teint en blonde (manifestation visible qui déclare son allégeance à Bobo/Baubô, l'oiseau jaune, sur le plan invisible latent) le narrateur nous dit que la mère d'Alma veut empêcher sa fille de sortir dans la rue. Le texte, quant à lui, nous indique que la mère invite sa fille à faire exactement le contraire. 
[...] the mother came out of her faint... "Alma," she said weakly, "Alma!" Then she said her husband's name several times, but neither of them paid any attention to her, so she got up without any assistance and began to take a part in the conversation. "Alma," she said, "you can't go out of this house until that hair of yours grows in dark again." (Williams 2012, 251)

Pourvu que notre lecture soit sensible aux ruses de Williams, nous reconnaissons un ordre dissimulé formulé par les prénoms, évoqués sans être produits, de la fille et du père. Increase, le prénom du mari, prononcé après celui d'Alma endosse une valeur de verbe à l'impératif : Alma increase, increase, increase! Sa mère, sans s'en rendre compte, exhorte sa fille à assumer la destinée inscrite dans les différents sens de son nom. Présentée par voix narrative, si on la transforme en discours cette phrase s'avère être performative (J. L. Austin). Elle fonctionne comme une formule magique. La suite de la nouvelle illustre le pouvoir des mots à agir sur la "réalité»; cet ordre modifie l'identité du sujet. Sur le plan de l'intrigue manifeste, il augure le « devenir prostituée » pour Alma; sur le plan autobiographique de l'intrigue latente, le «devenir dramaturge » pour Tom. Le même récit nous raconte ainsi deux histoires.

La stratégie de condensation fait que les nouvelles s'offrent à une lecture sur deux plans si distincts qu'ils peuvent même évoluer à l'opposé l'un de l'autre : le premier manifeste, ramassé, contrôlé, relevant d'une thématique sociale, le second invisible, « latent ", relevant de l'écriture de soi, libéré, libre, libertin et comique, fidèle à l'esprit poétique épicé de l'ancienne comédie attique telle qu'elle se manifeste chez Aristophane. Dans la partie suivante, nous verrons que la matière autobiographique développée dans les nouvelles est reprise dans le théâtre sous ce format condensé, à travers une double énonciation qui ouvre une voie d'action parallèle et invisible pour nous mettre face à la figure de l'auteur - qui rit.

Les résonances entre les noms d'Alma Winemiller de Summer and Smoke, Alma Tutwiler de « The Yellow Bird » et Oliver Winemiller de «One Arm » indiquent une perméabilité entre les genres qui nous permet de traiter cette pièce et ces nouvelles sur la base d'un dénominateur commun: le rire. Alma Winemiller est sujette à des rires nerveux, associés à sa nature : "Out of nervousness and self-consciousness, she has a habit of prefacing and concluding her remarks with a little breathless laugh. This will be indicated at points, but should be used more freely than indicated. " (Williams 2000, $107,110)$ Oliver Winemiller représente son rire par un gros « HA-HA!!? » sur ce croquis comique, lumineux et « chaud» qui, on l'a vu, fait de lui un avatar d'Iacchos. Quant à Alma Tutwiler, elle est l'héroïne d'une des rares nouvelles de Williams où, au rythme rapide d'une farce narrativisée, le comique et l'humour ont libre cours. Enfin, les protagonistes des trois œuvres se rattachent par la thématique de la prostitution, choisie par Williams pour représenter, sur un plan symbolique et métatextuel, son métier de dramaturge. Il existe d'autres échos qui rapprochent ces textes; toutefois nous aborderons uniquement ceux qui nous informent sur l'origine et les usages du rire. Ils sont tous en rapport avec Oscar Wilde et créent un réseau de correspondances rhizomiques que l'on tâchera de mettre en évidence entre ces trois œuvres.

Summer and Smoke repose sur la trame et les personnages de «The Yellow Bird» mais son action se concentre sur la première transformation d'Alma de fille de pasteur en prostituée. La pièce évolue autour de deux personnages qui, ironiquement, échangent leurs places. Amoureuse de John Buchanan, son voisin, médecin play-boy et matérialiste, Alma Winemiller, fille de pasteur idéaliste et réservée, refuse de se donner à lui lorsqu'un soir d'été il lui en fait la demande d'une manière qui blesse sa pudeur. 
Suite à quelques mois d'agitation extrême, elle finit par s'offrir à lui qui, devenu idéaliste, décline l'offre et, ayant lui-même traversé une relation trop passionnée avec Rosa Gonzales, danseuse de flamenco dont le père, dans son ivresse, tue le père de John, préfère épouser Nellie, une ancienne élève d'Alma. Alma se tourne alors vers un étranger, un commis voyageur de passage, qui sera son premier amant. L'action se déroule au cours de la fin d'une saison, couvrant un été, un automne et un hiver pour que la pièce se termine avec la perspective du printemps, saison de renouveaux. Sa division en deux parties, intitulées " A Summer ", "A Winter ", suggère l'existence d'une dimension mythologique dans sa structure, par l'association des saisons, sous forme de corrélats objectifs, aux humeurs des protagonistes qui naviguent entre chaleur et glace, fumée et transparence, deux paradigmes symboliques trompeurs car en réalité ils se rejoignent. C'est l'humeur de Déméter, la terre-mère, triste lorsque sa fille descend aux enfers (automne et hiver) et joyeuse lorsqu'elle en remonte (printemps et été) qui est suggérée par cette division structurelle qui évoque ici, comme dans nombre d'autres pièces de Williams, le rapt de Perséphone (Thompson 47 passim). Ce même mythe se trouve aussi à l'origine des mystères éleusiniens (Homer 460-489). Ces mystères nous intéressent par la place que leurs rituels d'initiation accordent à la sexualité et au rire. $\mathrm{Au}$ cours des processions, les initiés honoraient Baubô et Iacchos, figures de rire nodales de ces mystères (Olender 21-22, Magnien 80-82). Si en passant de la nouvelle à la pièce le mode n'est plus celui de la farce, on retrouve néanmoins le rire dans les didascalies et dans les dialogues sous une forme étouffée, violente ou hystérique. On le retrouve enfin qui hante la poétique et la structure de l'action à travers la thématique du double (John parlera du « doppelgänger » d'Alma). Cette thématique se dédouble et se démultiplie pour ouvrir la voie à une lecture autobiographique de la pièce dont (presque) tous les personnages émanent de l'héroïne de la nouvelle et sont des facettes diffractées de la figure de l'auteur. C'est peut-être dans cet aspect autobiographique et inattendu de cette œuvre que réside son côté farce - une farce jouée au public.

Tourmentés par une vie intérieure intense, Alma et John, sont modelés comme des jumeaux - des jumeaux inversés. L'un est rongé par le feu: "my head's on fire» (Williams 2000, 620); l'autre par de la glace qui, à la fin, s'avère être du feu pris pour de la glace: «it was flame mistaken for ice» (Williams 2000, 637). Leur ressemblance différenciée et leur vie intérieure ${ }^{23}$ déclenchent la thématique du double et le symbolisme palingénésique du phénix. Il participe à la création du personnage de John dont l'entrée sur scène est annoncée par la formule métaphorique de Dusty: «Bright like a new silver dollar » (575). Ce qui associe ce personnage aux dollars dits « Morgan », frappés entre 1878 et 1921, dont le verso fait figurer l'aigle américain en position de phénix (cf. image ci-contre). 


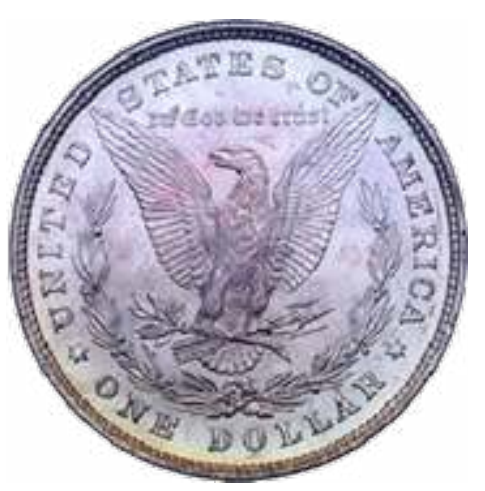

Dollar Morgan.

Photographie de l'auteur.

Le feu qui manque à cette représentation est évoqué dans les didascalies quelques lignes plus haut, lorsque John est qualifié de figure prométhéenne (Williams 2000, 575) - Prométhée est le titan qui a volé le feu aux dieux pour l'offrir aux hommes (Harvey 348). La chaleur et la fumée du titre suggèrent ce même symbolisme du phénix. Il se précise et se répercute à travers des décors et accessoires qui se rapportent aux palmiers, à la fumée, au feu, aux oiseaux et aux plumes. Pour aller vite, on peut mentionner quelques points qui véhiculent ce symbolisme en faisant écho aux deux nouvelles: la feuille de palmier utilisée comme éventail, puis détruite par Alma au début de sa transformation ("she crushes the palm-leaf fan », 601), reprend l'épisode similaire dans "The Yellow Bird " (supra 9); les cigarettes fumées par la mère d'Alma (596) reprennent cette même thématique de la même nouvelle; "La Golondrina " "4 (567), titre de la chanson d'Alma qui ouvre la pièce, fonctionne, par le biais de l'hétéroglossie, comme le plongeon d'Oliver dans les eaux de Palm Beach; elle indique d'emblée la fin heureuse de la pièce sur le « double invisible » du récit littéral - à savoir la régénérescence et le renouveau annoncés par les hirondelles qui arrivent au printemps ; le flamenco, dansé par Rosa devant John (618), contient la composante " flame » dans sa poétique et son étymologie le rattache au phénicoptère ${ }^{25}$ (ou flamant rose), en anglais flamingo ${ }^{26}$, qui fait de la maîtresse mexicaine de John une autre émanation du phénix; les plumes décoratives sur les chapeaux d'Alma et de sa mère (« wear a hat with a plume» Williams 2000,589 ) indiquent enfin l'engagement de ces personnages à cette même thématique ${ }^{27}$ (Williams 2000, 590, 596).

Le symbolisme palingénésique du phénix est omniprésent parce que, dans les nouvelles comme dans cette pièce, voire dans nombre de pièces de Williams, il constitue le moyen par lequel le dramaturge fait la transition entre l'écriture dramatique et l'écriture de soi, la transition d'une vision qui fait pleurer à une autre qui fait rire. Dans la section qui suit, nous verrons que cette transition se fait par une subtile réappropriation de structures, de thèmes et de personnages de l'œuvre d'Oscar Wilde que Tennessee Williams, l'auteur, choisit comme l'un de ses ancêtres littéraires. "The Yellow Bird" comme Summer and Smoke explore la symbolique du phénix pour raconter l'autobiographie de la figure de l'auteur Tennessee Williams.

Dans Summer and Smoke, la présence de Wilde est explicite: Alma cite une phrase de Lady Windermere's Fan (1891) prononcée par Lord Darlington ${ }^{28}$, dandy "réformé » par son amour pour l'héroïne éponyme, puritaine et idéaliste. Alma reprend cette phrase pour exposer l'horizon idéaliste de sa perspective sans savoir qui en est l'auteur : 
ALMA: How everything reaches up, how everything seems to be straining for something out of reach of stone-or human-fingers? To me-well, that is the secret, the principle back of existence-the everlasting struggle and aspiration for more than our human limits have placed in our reach... Who was that said that -oh, so beautiful thing! "All of us are in the gutter, but some of us are looking at the stars!"

JOHN: Mr. Oscar Wilde.

ALMA (somewhat taken aback): Well, regardless of who said it, it's still true. (612)

Cependant c'est la présence implicite de Wilde qui déclenche le rire. Systématiquement dissimulée, on la soupçonne derrière des répliques qui usent de l'esprit de repartie pour exercer une critique sociale. Williams n'est pas coutumier de cet usage qu'il emprunte à l'évidence à Wilde chez qui c'est une tactique systématique dans les comédies. Roger, personnage secondaire de Summer and Smoke, gentil et effacéé, montre des photos de sa mère jeune en costume de chasseresse à Alma :

ROGER : That is Mother in a hunting costume.

ALMA: [...] what was she hunting?

ROGER (gayly): Heaven knows what she was hunting. But she found Papa. (616)

7 Cette présentation du mariage comme jeu de chasse est courante dans les comédies de Wilde qui attribue le rôle du chasseur non aux hommes mais à des femmes comme la Duchess of Berwick dans Lady Windermere's Fan, ou Lady Backnell dans The Importance of Being Earnest. Cependant, ce ne sont pas tant les comédies de Wilde mais son roman, The Picture of Dorian Gray, puis ses deux pièces symbolistes, Salomé et La Sainte Courtisane or The Woman Covered with Jewels qui déteignent sur Summer and Smoke. On commencera à esquisser le rapport entre celles-ci à partir de l'entrée sur scène de Rosa Gonzales, au tout début de la première scène.

(As if brought by the courier of the tropics, Rosa Gonzales enters and crosses to the fountain. Her indolent walk produces a sound and an atmosphere like the gulf wind on the palmettos, a whispering of silk and a slight rattle of metallic ornaments. She is dressed in an almost outrageous finery, with lustrous feathers on her hat [...] a cascade of them, also diamond and emerald earrings.) (Williams 2000, 583).

8 Le son émis par le vent sur les feuilles des palmiers et la cascade de plumes sur sa coiffure, indiquent que l'arrivée sur scène de Rosa s'ancre dans la symbolique du phénix (par hétéroglossie et synecdoque). Les bijoux de Rosa évoquent la fin d'Alma dans «The Yellow Bird», lorsque son fils, John, lui en apporte tous les matins pour la rendre riche et lorsque son amant préféré, John lui aussi, lui recouvre le corps de joyaux au moment de sa consécration (Williams 2012, 255). Par ailleurs, danseuse de flamenco, Rosa évoque le sens de «danseuse " du prénom d'Alma de "The Yellow Bird» (supra p. 3 et Williams 1996, 252). Danseuse couverte de bijoux, Rosa est aussi dangereuse. Elle peut ruiner la carrière et la vie de John et, comme déjà mentionné, son père tue Dr Buchanan, père de John. Rosa s'apparente ainsi au type de la femme fatale. Ces aspects font écho à la figure éponyme du drame symboliste de Wilde, Salomé, rédigé en français en 1891, inspiré en partie par deux tableaux de Gustave Moreau, L'Apparition (1875) et Salomé dansant devant Hérode (1876), (Richmont-Garza 29, Russ 39): ces tableaux représentent Salomé couverte de joyaux. Plusieurs détails de l'action de Summer and Smoke s'inspirent de Salomé comme la thématique du double et la création des protagonistes comme des jumeaux. Comme John et Alma, Salomé et Iokannaan sont construits comme des jumeaux inversés (Clement 123). De même, sur le plan de l'onomastique, terrain que Williams exploite régulièrement, ce n'est pas seulement le prénom John qui évoque Jean-Baptiste de Salomé: le nom de famille Buchanan fait écho 
à Iocanaan, le Jean-Baptiste de Wilde. La danse de Rosa rappelle la danse des sept voiles de Salomé, y compris dans les détails. A la phrase obsessionnelle « Je baiserai ta bouche Iokanaan » de Salomé (Wilde 1993, 89, 91) fait écho celle que Rosa adresse à John «I want you - I, I want you!» (Williams 2000, 619). Les propos d'Hérode : «Dansez pour moi Salomé. Je vous donnerai tout ce que vous me demanderez...Tout ce que vous voudrez je vous le donnerai... " (Wilde1993, 133-135) sont repris dans "Anything that she want (sic) I get for her » de Papa Gonzales (Williams 2000, 620). Comme pour Dorian Gray de Wilde et Alma de la nouvelle (Williams 1996, 253), la vie dissolue menée par John avec Rosa n'affecte pas le physique de John. Toutefois, dans Summer and Smoke, la référence à Dorian Gray se "téléscope" avec la danse de Salomé avec une ruse qui dissimule le renvoi au chiffre sept du nombre des voiles : en regardant Rosa danser, John parle de son physique qui reste inaltérable en l'associant à ses sept costumes disponibles (si l'on se donne la peine d'en faire le compte) : "Did anyone ever slide downhill as fast as I have this summer? Ha-ha! Like a greased pig. And there isn't a sign of depravity in my face. And yet every evening I put on a clean white suit. I have a dozen. Six in the closet and six in the wash, and one on me. " (Williams 2000, 618-619). $\mathrm{Au}$ meurtre d'Oliver Winemiller qui, dans "One Arm ", associe le rire à la violence, fait écho le meurtre de Dr Buchanan par Papa Gonzales. Enfin, la structure chiasmatique de Summer and Smoke qui de manière ironique opère une permutation entre les protagonistes - la fille de pasteur devient courtisane et le play-boy médecin se dévoue, de manière idéaliste, à combattre le mal - fait écho à celle de La Sainte Courtisane or The Woman Covered With Jewels. Le titre de cette pièce inachevée de Wilde aurait pu être aussi celui de "The Yellow Bird» dont la fin présente la consécration d'une courtisane dont le corps est couvert de joyaux. La pièce de Wilde nous présente en effet la courtisane Alexandrine Myrhina et l'ermite Honorius qui se rencontrent à l'initiative de la première et décrivent chacun sa vie à l'autre. Leurs récits sont si convaincants qu'ils aboutissent à une double conversion: ironiquement, la courtisane reste dans le désert pour connaître l'amour de Dieu et l'ermite part à Alexandrie pour connaître les amours charnelles (Vernadakis 2009, 230-237).

On peut constater que certains traits d'Alma de «The Yellow Bird» sont redistribués à différents personnages de Summer and Smoke, notamment à Rosa et à John. Si Alma de "The Yellow Bird» est une représentation de la figure de l'auteur, John et Rosa mais aussi la mère d'Alma, Papa Gonzales, Dr Buchanan, Roger Doremus, Nellie et même Miss Bassett, sont aussi des parcelles identitaires de cette même figure, un personnage unique qui se démultiplie pour faire jouer aux uns et aux autres le rôle des différents prismes au travers desquels s'effectue la démultiplication. Cette logique de démultiplication fictionnelle du « je » de l'auteur se manifeste aussi dans The Picture of Dorian Gray : dans une lettre à Ralph Payne à ce sujet, Wilde écrit : "Basil Hallward is what I think I am: Lord Henry what the world thinks of me: Dorian what I would like to be - in other ages perhaps. » (Wilde, Letters, 585)

30 Le « je » démultiplié du dramaturge nous permet de déceler la formation d'un lien chez Tom Williams, l'auteur en herbe, entre le rire en tant que concept et l'œuvre de Wilde. Ce lien devient manifeste par la mise en contexte autobiographique d'un épisode fictionnel comique de Summer and Smoke. Il s'agit cependant d'un épisode qui repose sur une expérience violente, et sans doute traumatique. L'épisode comique se déroule dans le presbytère où Alma reçoit quelques amis « intellectuels ", dont Vernon, dramaturge en herbe à l'allure de Byron (Williams 2000, 598). Alma a également invité John qui, à 
l'étonnement de tous, a accepté d'y assister. Vernon doit donner lecture de sa pièce en vers, mais Alma ne veut pas que John trouve la réunion pénible. Mrs Bassett, personnage de farce, tout comme Vernon et Roger qui participent aussi à cet épisode, n'est pas là pour l'aider; bien au contraire. Ses interventions, comiques pour le public, créent une tension dramatique forte pour Alma.

ALMA: [...] Well, Now! We are completely assembled.

MRS. BASSETT (eagerly): Vernon has his verse play with him tonight!

ALMA (uneasily): Is that right, Vernon? (Obviously, it is. Vernon has a pile of papers

eight inches thick on his knees. He raises them timidly with downcast eyes.)

(Williams 2000,598)

L'épisode se termine en fiasco sans que la pièce ait été lue. Cependant, lorsque quelques semaines plus tard, Vernon a finalement lu sa pièce, Mrs Bassett fait le compte rendu suivant de sa réception :

MRS BASSETT: At the last meeting Vernon read his verse play.

ALMA: Oh! How was it received?

MRS BASSETT: Maliciously! Spitefully and vindictively torn to pieces like children

tear the wings of butterflies!

[...]

MRS BASSETT: But Vernon arose above it. There is nothing you can't rise above if you keep your courage. (Williams 2000, 629)

Pour que les propos de Mrs Bassett participent au récit latent, il faut encore avoir recours à l'hétéroglossie qui, par ricochet, nous conduit au prénom d'Alma, l'héroïne préférée du dramaturge (supra p.4 et Devlin 228). En effet, en grec butterfly se dit "psyché » et signifie à la fois papillon et âme. Ame, à son tour, se dit "alma »en espagnol : «My name is Alma, and Alma is Spanish for Soul» (Williams 2000, 537). Comme Alma et John, Vernon est, lui aussi, une parcelle identitaire de Tom, dramaturge en herbe. Quant au papillon, il est ici une métonymie de l'âme de Williams ${ }^{30}$. Sa mise en pièces pourrait correspondre à la part de violence qui participe au processus de métamorphose de la chenille en papillon, de Vernon ou de Tom à Tennessee Williams.

On peut cependant se demander quel est le rôle de Wilde dans cet épisode et dans ce processus et comment le lien entre Wilde et le rire se justifie, puisque jusqu'à présent il n'a été question ni de l'un ni de l'autre. Métonymie de l'âme de Williams, la pièce en vers descendue en flammes est aussi une métonymie pour une pièce en vers, bien précise, composée par Tom Williams. Car cet épisode fictionnel fait écho à un épisode réel : la lecture d'Ishtar, œuvre de jeunesse non publiée de Tom, devant deux de ses amis dont l'avis comptait pour lui. Dans son avant-propos à Candles to the Sun, William Jay Smith rapporte le souvenir suivant relatif aux échecs de Tom au début de sa carrière :

Tom Williams' next failure was a private rather than a public one. ...Tom read to Clark and me the just-completed draft of what was apparently his first attempt at poetic drama. It was Ishtar: A Babylonian Fantasy.... One would have left behind such attempts at Flaubertian exoticism, but no. Here presented to us in his rich Southern voice was a bit of Babylonian Gothic. ... he did not get very far with his reading before Clark and I exploded with laughter. (Williams 2004, XIV)

Il se trouve qu'Ishtar de Williams est un pastiche incontestable de Salomé de Wilde. Personnage composite chez Wilde, Salomé réunit en elle diverses figures mythologiques, dont la déesse de l'amour babylonienne, Ishtar. La formule « la danse des sept voiles " s'inspire d'ailleurs de la descente aux enfers de cette dernière et fait 
référence aux sept arrêts d'Ishtar, à chacune des portes des enfers, pour se défaire, à chaque fois, d'un article de sa parure (McCall, 117-126). La formule, La danse des sept voiles, a été inventée par Wilde pour signifier - toute proportion gardée - que la danse de Salomé conjugue art, plaisir et passion dans un strip-tease à la fois physique et métaphorique (Vernadakis 1989, 148-154). Les thèmes, les personnages, le ton, l'esthétique, les sources de Salomé de Wilde se retrouvent dans Ishtar de Tom, dont le manuscrit, conservé au centre Harry Ransom (Austin, Texas), nous permet de constater que la relation entre les deux est - pour ainsi dire - intime. Le Cantique des Cantiques qui, chez Wilde, est utilisé pour démasculiniser et déféminiser les personnages, constitue également le canevas sur lequel Williams projette des gros plans de la lune. Les deux œuvres se répondent ainsi de manière profonde.

SALOME: How good to see the moon! She is like a little piece of money [...] she is cold and chaste [...] HEROD: The moon has a strange look tonight [...] She is naked too. She is quite naked [...] Ah! Look at the moon! She has become red. She has become red as blood. (Wilde 62, 94, 138)

Pour faire écho aux propos de Salomé, Ishtar les inverse; cependant, dans le cas de reprise d'une œuvre de Wilde par Williams, l'inversion ne peut-elle pas faire partie de l'intention poétique de base? Si l'objectif est de faire rire, c'est possible.

THE BRIDE: Lo! The moon is not cold [...] Neither it is small and round. Like the silver coins that merchants jangle in their palms. The moon is heated with fountains of blood. [...] Behold the moon has fallen, she has touched the lake she has dabbed it with her toes like a naked girl. (Ishtar, Harry Ransom (Austin, Texas) box 21 , folder 10)

Dans l'apprentissage de son métier de dramaturge, Tom Williams commence par se réapproprier ce qu'il aime dans l'œuvre d'autrui. Cette attitude est implicitement représentée comme un acte sexuel dans "The Yellow Bird», sur le plan de l'action autobiographique latente, à travers le vécu fictionnel d'Alma dans l'épisode suivant :

[...] in her miserable little furnished room-or crib, as it actually was- [...] Tickets from pawnshops were stuck round the edge of the mirror along with many, many photographs of young men, some splitting their faces with enormous grins while others stared softly at space (Williams 2012,254).

Les photographies des amants évoquent des masques de comédie et de tragédie qui bordent le miroir où elle se regarde chaque matin. Ce qui évoque l'analogie entre l'évolution d'Alma et celle de Tom qui se projette dans la personne du dramaturge à venir Tennessee Williams. Les tickets des bureaux de prêteurs sur gage, intercalés entre les photos/masques de théâtre, peuvent aussi être interprétés comme les engagements d'Alma/Tom dans son processus de devenir dramaturge. C'est d'abord un engagement sentimental pour Alma, intellectuel pour Tom, face aux «amants» / écrivains fréquentés au cours de ses apprentissages. C'est ensuite un engagement de probité et commerçant, face aux prêteurs, aux agents artistiques ${ }^{31}$, aux journaux et revues qui font confiance à Tom et publient ses histoires ou tentent de promouvoir ses pièces, aux membres du jury de la fondation Rockefeller qui lui avaient attribué une bourse en 1939. Ishtar pourrait être vue comme l'un de ces tickets de " pawnshops » qui entourent le miroir d'Alma, et la lecture de cette pièce par Tom à ses amis, une tentative de laisser au mont-de-piété un objet de valeur créé par contact avec un amant. La sexualité qui fait rire se mêle ici à l'autobiographie par le biais de la phonétique : la voix de Tom Williams, marquée par ce riche accent du Sud, dont parle Jay Smith dans la citation plus haut, pourrait bien associer l'art de l'écriture dramatique et la prostitution par cette homophonie, ou presque, entre " pawnshops " 
et «porn-shops ». Certes, dans les années 1940, il n'existe pas des porn-shops ; le terme " porn " néanmoins existait. Williams, dont l'œuvre fait écho à l'ancienne comédie attique par le nombre d'exemples de poétique scatologique qui sert le rire, pourrait imaginer l'espace littéraire comme un espace d'échange «libre ». Après tout, l'image qu'il emploie pour l'auteur en herbe est celle d'une prostituée ${ }^{32}$. Si l'on ne rit pas avec l'image d'Alma qui se regarde dans ce miroir tous les matins pour constater que son visage ne porte ni les marques de sa vie dissolue ni celles du temps, on rit lorsque derrière l'évocation de Dorian Gray" ${ }^{33}$ on reconnaît l'association "commerciale » de l'homosexualité des deux écrivains sous l'égide de deux autres homophones - ou presque - Bobo ${ }^{34}$ et Baubô, l'oiseau jaune des procès mystérieux de Salem et la vieille nourrice des processions initiatiques aux mystères éleusiniens. Auquel cas, le fils d'Alma pourrait être une représentation d'Iacchos, dieu du rire et porteur de lumière ${ }^{35}$. C'est aussi une nouvelle représentation figurative et métaphorique de l'œuvre - de l'enfant - de Williams.

Pour revenir à la lecture de Vernon, il convient de voir dans cet épisode une passerelle entre réalité et fiction sur laquelle Ishtar et Salomé jouent un rôle médiateur important : toute proportion gardée, en dévoilant sa création à ses amis Clark et Smith, Tom laisse transparaître une partie de son intimité - son âme. Seulement, dans les années 1940, l'œuvre de Wilde n'est plus d'actualité : l'intérêt de Tom pour l'idéalisme et son goût pour des formes d'art surchargées comme le symbolisme, l'orientalisme et la décadence, sa passion pour les mythes extravagants, son attrait pour l'esthétique baroque, bref, pour les matériaux que Wilde intègre dans Salomé et La Sainte Courtisane à la toute fin du XIX ${ }^{e}$ siècle, ne passent plus dans les années 1940. A l'instar de ses amis, l'Amérique, réceptrice de l'œuvre de Williams, n'aurait accueilli ces tendances que par un rire moqueur de déni. Ce déni fait le lien avec l'homosexualité de Williams et de Wilde: les aspects esthétiques que Williams apprécie chez Wilde et qui avaient été acceptés dans le passé, sont alors rejetés parce qu'ils ne sont plus de son temps - tout comme son homosexualité. Williams engage ainsi des stratégies de détour. Du coup, il y a une confrontation permanente entre l'auteur et le public. Ce qui transforme chaque acte de création en acte de violence qui doit finir par le triomphe de l'auteur. Ce triomphe se traduit par un rire.

Le rire invisible qui hante les pièces de Tennessee Williams est la riposte de Tom au rire explosif de ses amis. Il émane d'une âpre transformation de sa vulnérabilité en force, de sa fragilité en provocation et, toute proportion gardée, en violence. C'est le rire de l'auteur qui, à travers des stratégies adaptées, parvient, malgré la résistance de ses amis - la résistance du public - à faire passer dans son œuvre tout ce qu'il veut, sans faire rire personne pour autant - si ce n'est lui-même.

Ce type de rire est partagé uniquement entre intimes ou - pour ne pas perdre de vue le contexte des mystères d'Eleusis qui cadre nos réflexions - entre « initiés ». On retrouve un rire analogue dans la comédie de Wilde The Importance of Being Earnest, pièce dans laquelle Wilde s'affronte à "earnestness", l'idéal le plus important des Victoriens. Jusqu'aux années 1990, la pièce se donnait sans que personne ne se soit avisé de la dimension homosexuelle du jeu de mots du titre : le prénom Ernest qu'endossent les deux protagonistes désigne aussi l'idéal de sérieux, earnest(ness). Cependant, Ernest est aussi une paronomase d'Uranist, «homosexuel » (Bristow, 18), détail qui éclaire une avalanche de sous-entendus et allusions, souvent scatologiques, d'inspiration autobiographique (Aquien 105). Earnest s'offre ainsi à une lecture autobiographique 
(Drugeon, Vernadakis 49) qui permet à Wilde de se rire de tout, y compris de son homosexualité qu'il « affiche » derrière le voile de ses jeux de mots d'onomastique.

Williams adopte la même attitude, à la fois secrète et provocatrice, pour révéler par condensation sémantique et dévoilement des traits identitaires que la société rejette. Comme on l'a vu avec l'exemple de Stanley dans A Streetcar Named Desire et comme il le fait dans Summer and Smoke où John, par exemple, est amateur de "cock-fights " organisés par Papa Gonzales (Williams 2000, 610), la sexualité est toujours dévoilée derrière le rire. Suscité par un anasyrma, le rire exige la connivence du public pour que l'objet sexuel qui le déclenche soit révélé.

A l'inverse de Williams, personne n'a reproché à Wilde son idéalisme - à l'exception de lui-même. En revanche, la révélation de son homosexualité qui, aujourd'hui, dans Earnest nous fait rire, lui a valu un procès, deux ans de travaux forcés et la fin de sa carrière de dramaturge. La nouvelle "The Yellow Bird » commence avec le procès que les puritains font à une ancêtre d'Alma, déclarée sorcière à Salem parce qu'elle fréquentait Bobo, l'oiseau jaune, messager du diable.

The first American progenitor had settled in Salem, and around him and his wife, Goody Tutwiler, née Woodson, had revolved one of the most sensational of the Salem witch trials. Goody Tutwiler was cried out against by the Circle Girls.... They claimed that Goody Tutwiler ... had appeared to them with a yellow bird which she called by the name of Bobo and which served as interlocutor between herself and the devil to whom she was sworn. ... Goody Tutwiler was accordingly condemned and hanged, but this was by no means the last of the yellow bird named Bobo, it had manifested itself in one form or another, and its continual nagging had left the Puritan spirit fiercely aglow, from Salem to Hobbs, Arkansas, where the Increase Tutwiler of this story was preaching. (Williams 2012, 248)

Il est fort probable que l'ancêtre d'Alma pendue à Salem soit une référence oblique à Wilde, dans le processus d'adoption d'ancêtres du dramaturge en herbe, Tennessee Williams. Wilde a aussi été condamné pour avoir osé exposer au rire de tous l'idéalisme puritain qu'il télescope avec son homosexualité. Dans ce cas, Bobo, l'oiseau jaune, serait l'esprit régénérateur du rire que Wilde a mis à l'honneur par cette révélation - un anasyrma qu'il entreprend en se faisant lui-même un avatar de Baubô. Iacchos, c'est l'enfant d'Alma, l'œuvre de Williams, qui éclaire le côté de l'humain gardé voilé, sa sexualité qui, une fois révélée, fait rire. Par condensation syncrétique, l'esprit de Baubô comporte la symbolique palingénésique du phénix qui, comme le désir, se renouvelle de ses cendres.

A la fin de Summer and Smoke, Alma finit par rire de manière naturelle; toutefois, cela se fait uniquement après qu'elle aura procédé à un anasyrma : "Alma pushes her veil back with an uncertain gesture. » (Williams 2000, 640) Il s'agit, certes, d'une exposition non pas de son sexe, mais de son visage; toutefois ce qu'elle relève, son voile, "veil», la rattache encore à la performance de Salomé qui se déshabille en dansant (cf. Dance of the seven veils), et par ce biais, aussi à Ishtar. Le dialogue qu'elle engage, en partie en espagnol, avec le jeune commis-voyageur, préconise, d'ailleurs, que ce premier anasyrma peut en cacher un autre. Et la révélation, cette fois, est plus drôle, plus radicale aussi. Elle s'apparente à un coming-out :

THE YOUNG MAN (delightedly): Ha ... ha ... ha ! Sometimes un poquito is plenty! (Alma laughs ... in a different way than she has ever laughed before, a little wearily, but quite naturally.) What's there to do in this town after dark?

ALMA: There's not much to do in this town after dark, but there are resorts on the lake that offer all kind of after-dark entertainment. There's one called Moon Lake 
Casino. It's under new management, now, but I don't suppose its character has changed.

THE YOUN G MAN: And what was its character?

ALMA: Gay, very gay... (Williams 2000, 642)

défaite pour révéler en profondeur l'ineptie de ce que l'on nous apprend à respecter « et dévoiler la vérité cachée, à la fois ridicule et sordide, qui se retrouve derrière les valeurs que l'on nous présente comme les plus incontestables " (Badiou 17). Insaisissable (ou presque), il retentit dans les choix de mots ambivalents et glissements de la langue qu'ils produisent pour les recontextualiser de manière à ce qu'ils signalent un sens implicite sexuel. Bergson, dont Williams avait étudiée l'œuvre en 1936 à l'Université de Washington, dans la conclusion de son traité sur le rire (1900), a recours à l'image : il compare le rire à l'écume des vagues (Bergson 153). Ce qui associe le déclenchement du rire à la naissance d'Aphrodite « née de l'écume ».

C'est ainsi que des vagues luttent sans trêve à la surface de la mer, tandis que les couches inférieures observent une paix profonde. Les vagues s'entrechoquent, se contrarient cherchent leur équilibre. Une écume blanche, légère et gaie, en suit les contours changeants. Parfois le flot qui fuit abandonne un peu de cette écume sur le sable de la grève. L'enfant qui joue près de là vient en ramasser une poignée, et s'étonne l'instant d'après, de n'avoir plus dans le creux de la main que quelques gouttes d'eau, mais d'une eau bien plus salée, bien plus amère encore que celle de la vague qui l'apporta. Le rire naît ainsi que cette écume. Il signale, à l'extérieur de la vie sociale, les révoltes superficielles. Il dessine instantanément la forme mobile de ces ébranlements. Il est, lui aussi, une mousse à base de sel. Comme la mousse, il pétille. C'est de la gaieté. Le philosophe qui en ramasse pour en goûter y trouvera d'ailleurs quelquefois, pour une petite quantité de matière, une certaine dose d'amertume. (Bergson 152-153)

Cette écume, qui dans le texte d'Hésiode peut provenir de la lutte des vagues ou bien du sexe mutilé de Cronos (Hésiode 39-40), indique qu'il existe un rapport entre le rire et le désir, le rire et la sexualité, la violence et la mort. C'est ce rapport que Williams explore à travers l'anasyrma de Baubô pour faire écho aux derniers mots de Salomé de Wilde : « le mystère de l'amour est plus grand que le mystère de la mort. Il ne faut regarder que l'amour » (Wilde 1893, 163) - et en rire.

\section{BIBLIOGRAPHIE}

Abirached, Robert. « Comédie ». Dictionnaire des genres et notions littéraires. Encyclopaedia universalis. Paris : Albin Michel, 1997 ?

Aquien, Pascal et Xavier Giudicelli (dir). The Importance of Being Earnest d'Oscar Wilde. Paris : PUPS, 2014. 
Austin, J. L. How to Do Things with Words (1962). Cambridge MS: Harvard University Press, 1975.

Badiou, Alain, avec Nicolas Truong. Eloge du théâtre. Paris. Flammarion : 2013.

Bergson, Henri. Le Rire, Essai sur la signification du comique (1900). Paris, PUF, 1962.

Brian, Peters. «Queer Semiotics of Expression: Gothic Language and Homosexual Destruction in Tennessee Williams's “One Arm" and "Desire and the Black Masseur" ». The Tennessee Williams annual Review, 8 (2006). Web.

Bristow, Joseph (ed.). The Importance of Being Earnest and Related Writings. London: Routledge, 1992.

Calimach, Andrew. Lover's Legends. The Gay Greek Myths. New York: Haiduk Press, 2002.

Clément d'Alexandrie. Discours aux Gentils. (Le Protreptique) Traduit par Eugène de Genoude. (Les Pères de l'Eglise). Paris : A Royer, Editeur, 1843.

Clément, Catherine. "Désir de Sainte”. Strauss Salomé. L'Avant-scène opéra 47-48, (Janvier-février 1983) pp. 123-127.

Cox, Davis. "Tickling rats and giggling dolphins: do animals have a sense of humour?" The Guardian, 17 nov. 2015. Web. Consulté le 31-09-2018.

Daremberg et Saglio (1877). Dictionnaire des antiquités grecques et romaines. http://dagr.univtlse2.fr/. Web. Consulté le 31-09-2018.

Débusscher, Gilbert, « Creative Rewriting European and American influences on the dramas of Tennessee Williams." In Matthew Roudané, The Cambridge Companion to Tennessee Williams. Cambridge: CUP, 1997, pp. 167-188.

Devlin, Albert J. (ed.) Conversations with Tennessee Williams. Jackson and London: University Press of Mississippi, 1986.

Drugeon, Marianne et Emmanuel Vernadakis. Oscar Wilde. The Importance of Being Earnest. Neuilly sur Seine : Atlande, 2014.

Frazer, Georges, James. The Golden Bough, a Study in Magic and Religion. Abridged ed. New York. Macmillan, 1922.

Fraser, Kathleen. Before They Were Belly Dancers. European Accounts of Female Entertainers in Egypt, 1760-1870. Jefferson : McFarland, 2015.

Genette, Gérard. Métalèpse. De la figure à la fiction. Paris : Seuil, 2004.

Grimal, Pierre. Dictionnaire de la mythologie grecque (1951). Paris : PUF, 1986.

Hartnoll, Phyllis (ed.). The Oxford Companion to the Theatre (1951). London : OUP, 1976.

Harvey, Paul. The Oxford Companion to Classical Literature (1937). Oxford : 1984. OUP. Print.

Homer. Hymnes, Texte établi et traduit par Jean Humbert. Paris : Les Belles Lettres, 1997.

Hésiode. Théogonie ; Les travaux et les jours ; Le bouclier. Paris : Les belles lettres. 1996.

Kakutani, Michiko. «The Legacy of Tennessee Williams », The New York Times on the Web, 06-03-1983. Web. Consulté le 31-09-2018.

Lahr, John. Tennessee Williams. Mad Pilgrimage of the Flesh. London: Bloomsbury, 2015.

Lawrence, D. H. Lady Chatterley's Lover [1928]. Wordsworth Editions. N.d. Print.

Letissier, Georges. Rewriting/Reprising: Plural Intertextualities. Newcastle upon Tyne : Cambridge Scholars, 2009. 
Magnien, Victor. Les mystères d'Éleusis: leurs origines, le rituel de leurs initiations. Paris : Payot, 1929.

May, Charles, E. The New Short Story Theories. Athens: Ohio University Press, 1994.

McCall, Henriette. Mythes de la Mésopotamie. Paris : Seuil (Points), 1994.

Montague, Rhodes, James. The Apocryphal New Testament: Being the Apocryphal Gospels, Acts, Epistles, and Apocalypses [1924]. Oxford : Clarendon Press, 1980.

Mpoukalas, Pantelis. “Aristophane, Euripide et le dème des femmes athéniennes. Les Thesmophories comme comédie du retournement." dans Aristophane, Les Thesmophories. Athènes : Festival d'Athènes et d'Epidaure 2018, 2018.

O'Connor, Jacqueline, « The "Neurotic Giggle": Humor in the Plays of Tennessee Williams». Studies in American Humor, New Series 3, 6, Special Issue: Recovering New Humor (1999). Pennsylvania: Penn State University Press, 1999, pp 37-48.

Olender, Maurice. « Aspects de Baubô : textes et contextes antiques ». Revue de l'histoire des religions, vol. 202, 1985, pp 3-55.

Plutarque, Traité de la vertu des femmes, dans, Traités. Cuvres Morales, t. 7, V, 1. Paris : Les belles lettres, 1984.

Richmont-Garza, Elizabeth. "The Double Life of Salomé: Sexuality, Nationalism and SelfTranslation in Oscar Wilde”, in Michael E. Bennett. Refiguring Oscar Wilde's Salomé. Amsterdam: Rodopi, 2011.

Russ, Andrew R. “Wilde's Salomé: The Chastity, Promiscuity, and Monstrosity of Symbols" dans Michael E. Bennett. Refiguring Oscar Wilde’s Salomé. Amsterdam: Rodopi, 2011.

Saddic, Annette J. Tennessee Williams and the Theatre of Excess: The Strange, the Crazed, the Queer : Cambridge, CUP, 2016.

Smith-Howard, Alycia, and Greta Heintzelman. Critical Companion to Tennessee Williams. A literary Reference to His Life and Work. New York: Facts on File, Inc. 2005.

Thompson, Judith J. Tennessee Williams' Plays. Memory, Myth and Symbol. New York: Peter Lang, 2002.

Urban Dictionary, https://www.urbandictionary.com/ web.

Vernadakis, Emmanuel. "Baubô", dans Pierre Brunel, Dictionnaire des mythes Féminins. Paris : Editions du Rocher, 2000, pp. 229-236.

-- Gomez Carrillo. Enrique. L'évangile de l'Amour. Paris : Les Introuvables, L'Harmattan, 2003.

-- "Oscar Wilde, Tennessee Williams and the Palimpsest of the Courtesan", dans Georges Letissier. Rewriting/Reprising: Plural Intertextualities. Newcastle upon Tyne : Cambridge Scholars, 2009, pp. 229-241.

--. Le prétexte de Salomé, pour une approche de l'œuvre d'Oscar Wilde (thèse de doctorat en deux volumes). Paris : Université de Paris VII, Institut d'Anglais Charles V, dec. 1989.

-- « Les noms du désir dans A Streetcar Named Desire », in Coup de théâtre 19/ numéro spécial Tennessee Williams et Un tramway nommé Désir. Paris : RADAC, 2003 (p 66-81).

Webster's Revised Unabridged Dictionary [1913]. http://www.webster-dictionary.org/ web.

Wilde, Oscar. Salomé (1893). Paris : GF Flammarion, 1993.

--. The Complete Letters of Oscar Wilde. Edited by Merlin Holland and Rupert Hart Davis. London :

Fourth Estate, 2000. 
--. The Complete Works. London : Harper and Row. 1989.

Williams, Tennessee. Collected Stories (1985). London : Vintage, 2012.

--. Ishtar. Harry Ransom (Austin, Texas), box 21, folder 10.

--. Notebooks, edited by Margaret Bradham Thornton. New Haven and Lindon : Yale University

Press, 2006

--. Period of Adjustment, Summer and Smoke, Small Craft Warnings. New York : Penguin, 1982.

--. Plays, 1937-1955. New York : The Library of America, 2000.

Windham, Donald (ed.). Tennessee Williams' Letters to Donald Windham, 1940-1956. New York : Holt, Rinehart and Winston, 1977.

\section{NOTES}

1. Plusieurs critiques se sont penchés sur l'humour des pièces de Williams mais le rire, même s'il est abordé par certains, n'a pas encore constitué un sujet d'étude à part. Jacqueline O'Connor insiste sur son importance dans "The "Neurotic Giggle": Humor in the Plays of Tennessee Williams». Plus récemment, Annette J. Saddic l'associe à l'horreur pour l'aborder par le biais de l'excès dans Tennessee Williams and the Theatre of Excess: The Strange, the Crazed, the Queer.

2. Peters analyse ces images dans le cadre du sadisme et du masochisme, concepts qui ne sont pas intégrés dans cette étude.

3. Comme par exemple l'ouvrage volumineux de G. Frazer, The Golden Bough (Vernadakis 2003, 66-67).

4. "Williams was a man of feeling rather than intellect; an artist, not a scholar" (Debusscher 187).

5. Ainsi, les femmes perses retroussent leur vêtement pour renvoyer leurs fils et leurs époux sans courage au combat (Plutarque 89), et les femmes lyciennes ont recours au même procédé pour faire fuir Bélérérophont et faire en même temps reculer le raz-de-marée qui les menace (Plutarque 115).

6. "Yet each man kills the thing he loves", The Ballad of Reading Gaol [1897], (Wilde 1989, 843).

7. Les comics sont convoqués sous forme graphique ou cinématographique. Dans « The Yellow Bird », le fils d'Alma qui, à peine né, se rend chaque matin à la mer en rampant (Williams 2012, 255), emprunte les traits de Swee'Pea, fils de Popeye le marin, créé en 1929 par Elzie Crisler Segar. Dans Summer and Smoke, Papa Gonzales, caricature grotesque du Mexicain stéréotypé, évoque Speedy Gonzales de Robert McKimson. Tom, dans The Glass Menagerie, se fait l'écho de Tom the Alley Cat. Derrière Blanche et Stanley dans A Streetcar Named Desire on reconnaît la relation entre Tweetie et Sylvestre - Blanche est comparée par Stanley à un canari (Williams, 2000, 530). Il se peut que les comics rattachent ici le rire aux traits du trickster.

8. Rosa de Summer and Smoke, Esmeralda de Camino Real se définissent comme danseuses; nous verrons qu'Alma de "The Yellow Bird» et de Summer and Smoke et John Buchanan de la même pièce sont associés à la danse des sept voiles. Il en est de même pour Stanley, Stella et Blanche de Streetcar, ainsi que l'indique la dernière phrase de la pièce, «STEVE : This game is a seven-card stud. » (Williams 2000, 226), évocation « voilée » de cette danse par les sept cartes tirées.

9. De l'Angleterre aux Etats-Unis, du XVIe siècle à l'éternité, des ancêtres européens et américains d'Alma à la béatification de celle-ci.

10. Voir, par exemple, la chronologie proposée dans The Cambridge Companion to Tennessee Williams (Roudané XVI-XXIV).

11. Alma de Summer and Smoke souffre de palpitations comme Williams vers la fin des années 1930 (Notebooks 111) et prend calmants et somnifères, comme lui, pour dormir (Notebooks 99, 113). 
Alma de la nouvelle occupe le grenier - the attic - de la maison parentale, comme Tom lorsqu'il habite encore chez ses parents (Notebooks 101, 107, 169). De même, on trouve bien, dans les virées en voiture d'Alma de la nouvelle, quelque chose de ce que Tom décrit de ses sorties en voiture avec Joseph Safra (Notebooks 117-119).

12. Bien évidemment, il existe des pièces (Shakespeare, Claudel etc.) qui durent plus longtemps. Toutefois, les metteurs en scène les abrègent pour que le temps de la représentation ne dépasse pas les limites « raisonnables » imposées par les usages et la disponibilité du public.

13. Alma : danseuse orientale, comme déjà vu.

14. Il indique aussi le rapport étroit entre Alma et l'héroïne de A Streetcar Named Desire, Blanche DuBois dont le nom de femme mariée est Blanche Gray (le mari défunt se nommait Allan Gray, Williams 2000,490 ) et qui refuse de vieillir. Blanche imagine sa mort se dérouler comme celle d'Alma de la nouvelle, au milieu de l'océan, sous le regard langoureux d'un jeune et beau docteur de la marine (Williams 2000, 559) en fusionnant ainsi deux expériences distinctes, éros et thanatos.

15. Cette scène est inspirée de la vie que Williams menait, pas à la Nouvelle Orléans, mais à Santa Monica, en Californie, en septembre 1943 - a moment où il découvrait l'œuvre de Wilde. Voir la description que Williams fait de sa chambre dans une lettre à Donald Windham datée du 20 septembre 1943. Il dit, par exemple, "The ants are literally taking over my place. I have left the dishes unwashed for about ten days. When I need a clean dish I just turn on the faucet and let one of them rinse off. » (Windham, 104-105).

16. Voir l'article de David Cox dans The Guardian du 17 novembre 2015, "Tickling Rats and Giggling Dolphins: Do Animals Have a Sense of Humour?" ainsi que des très nombreux clips vidéo sur You Tube sur des dauphins qui rient.

17. Voir par exemple sur le site Lovethispic, l'une des images disponibles montrant des dauphins qui rient : http://www.lovethispic.com/uploaded_images/28975-Laughing-Dolphins.jpg

18. Voir François Lenormant « Descente aux enfers de Dionysos», dans Daremberg et Saglio, Le Dictionnaire des Antiquités grecques et romaines (1877), https://www.mediterranees.net/civilisation/ religions/dionysos/bacchus7.html

19. Williams fait référence aux toiles de Van Gogh dans ses œuvres et dans ses carnets, voir par exemple, Notebooks 70, 113, 189 etc.

20. Ah yes, to be passionate like a Bacchante, like a Bacchanal fleeing through the woods, to call on Iacchos, the bright phallos that had no independent personality behind it, but was pure godservant to the woman!

21. Le rapport de Laura à l'œuvre de Williams est métonymique. Il devient manifeste par le symbolisme de son prénom qui évoque la plante sacrée d'Apollon, celle qui couronne les têtes des poètes « lauréats » aux concours dramatiques de l'antiquité.

22. Voir par exemple, l'entrée du 29 avril 1938 dans les carnets de Williams : « What do I want ? I want love and creative power! - Eh bien! Get it! (Notebooks 117).

23. Qui par ailleurs évoquent le côté fantastique de la nouvelle puisqu'il est question de doppelgänger (Williams 2000, 582, 583, 588).

24. Qui, en espagnol, signifie « l'hirondelle».

25. Il faut cependant noter que cette étymologie du flamenco est incertaine. Pour certains, elle s'enracine dans les termes arabes felah-menkoub (" paysan errant »), pour d'autres dans le nom d'un couteau, ou encore dans l'origine flamande des gitans ou leur allure flamboyante. http:// www.flamenco-classical-guitar.com/historique-flamenco.php

26. Ce qui crée aussi des passerelles avec A Streetcar Named Desire par l'évocation de l'hôtel « Flamingo » à Laurel, [cf. « go in flame »] où Blanche commence à se " consumer » (Williams 2000, 530) avant d'évoquer explicitement le feu pour se faire " purifier " - ou renaitre - en se débarrassant de Mitch qui tente de lui faire l'amour (548) et se préparant à recevoir le viol de Stanley qui, aussi absurde que cela puisse paraître, marquera sa renaissance. 
27. Ils indiquent aussi le retour vers un passé réculé, avant la Réforme, plus libre ou libertin, recherché par l'auteur, comme il l'annonce dans « The Yellow Bird » par le « saut » accompli par Alma pour retrouver ses racines : «when [Alma] was alone (...) it sometimes seemed as if she weren't alone, as if someone were with her, a disembodied someone, perhaps a remote ancestor of liberal tendencies who had been displeased by the channel his blood had taken till Alma kicked over the traces and jumped right back to the plumed hat cavaliers » (237). Notons que le sens du prénom d'Alma en grec, $\alpha \dot{\lambda} \mu \alpha$, est « saut ».

28. "We are all in the Gutter but some of us are looking at the stars". Oscar Wilde, Lady Windermere's Fan, acte 3.

29. Roger de Summer and Smoke a pratiquement la même fonction face à Alma que Mitch face à Blanche dans A Streetcar Named Desire.

30. L'image du papillon se retrouve aussi dans les didascalies au tout début de A Streetcar Named Desire pour décrire Blanche : "There is something about her... that suggests a moth » (Williams $2000,471)$.

31. Déjà en mars 1939, lorsqu'il remporte le prix spécial au concours dramatique du Group Theatre, Williams reçoit des propositions d'agents artistiques pour l'accompagner (Leverich 296-299). Lorsqu'il s'engage avec Audrey Wood, il lui écrit régulièrement sur ses projets sous forme de propositions ou de promesses (par ex. voir dans Notebooks 156, note 259).

32. On peut se rappeler que la « crèche » d'Alma/Williams-auteur-en-herbe, se situe rue Bourbon, voie royale (cf. Camino Real) pour devenir dramaturge. La dé-composition phonique du nom de la rue constitue un jeu de mot scatologique qui suit la logique du « pawnshop/porn-shop ».

33. Une ligne plus bas dans ce même passage (voir supra p.6).

34. C'est probablement dans un poème de Gertrude Stein que Tennessee Williams a rencontré le non Bobo. Pour féliciter son protégé Robert (Bob) Haas de son projet de mariage avec Louise Krause, amie de Tennessee Williams, Stein compose un petit poème qui contient un jeu de mots. Elle se sert du nom d'un passereau chanteur appelé bobolink (en Français goglu des prés) qu'elle décompose pour signifier l'union du couple : «Louise and Bob-o-link/They are to be married». Louise, avait décoré son sapin de Noël avec des copies de ce petit poème qu'elle a également lu devant ses amis - dont Tennessee Williams- le jour de Noël pour leur annoncer son mariage avec Bob. (Notebooks 178, note 295).

35. Sa naissance dans la nouvelle s'associe à différentes déclinaisons du chiffre 666, et par cela à la figure satanique de l'Antéchrist: la transformation d'Alma commence six mois après un épisode mémorable à midi et demi $(6+6):(6+6+6+6+6)$, lorsque celle-ci a 30 ans $(30=6+6+6+6+6)$ alors que John vient au monde lorsqu'Alma a 36 ans $(6+6+6+6+6+6)$. L'heure de départ du fils pour aller chercher des trésors marins est $6 \mathrm{~h} 30$, c'est-à-dire $6:[6+6+6+6+6]$ etc. (Vernadakis 2013, 56) On se souviendra, par ailleurs, dans A Streetcar Named Desire du rapport de Stanley à la lumière au cours de sa nuit de noces où il casse les ampoules électriques avec le talon d'une pantoufle de Stella (Williams 2000,505) pour déclarer ainsi son lien à Lucifer, l'ange déchu de lumière. Il se rattache à lui par paronomase, étant systématiquement appelé Stan par ses proches - cf. Satan. Le prénom de Stella, qui signifie " étoile » en italien, rattache aussi cette dernière à Iacchos, " l'astre lumineux » des Mystères selon Aristophane (supra, p. 8). Résidents du quartier «Elysian Fields » - où l'on se rend par changement à la station "Cimetières » (Williams 2000, 471) - Stella et Stanley sont, comme Iacchos, des figures chtoniennes de lumière, qui font rire par dévoilement de ce qui est d'habitude caché, propre à l'univers d'Hadès, dont le nom signifie, l'invisible. 


\section{RÉSUMÉS}

Je me propose d'explorer la façon dont le rire se manifeste dans le théâtre de Tennessee Williams entre 1941 et 1948, à partir d'une pièce, Summer and Smoke (1948), et de deux nouvelles rédigées en parallèle, "The Yellow Bird » (1941-1947) et " One Arm » (1942-1948). Il sera aussi question, dans une moindre mesure, de The Glass Menagerie (1945) et de A Streetcar Named Desire (1947). Cette étude nous permettra de considérer que ces pièces, voire l'ensemble du théâtre de Williams, comportent ou suscitent un rire ambivalent mais salvateur. Invisible ou presque, ce rire travaille les intrigues, habite les personnages et vagabonde comme un doppelgänger entre les mots et les phrases pour éclairer ce qui parait sombre et démystifier ce qui semble poignant. Dans le présent article, je tenterai de montrer que les pièces de Williams ne sont pas ce qu'elles semblent être. Imposant, le rire qui les hante finit par drainer le pathos qui s'en dégage au profit d'une ironie spirituelle et salutaire.

I propose to look at laughter in the theater of Tennessee Williams between 1941 and 1948 through a close study of a single play, Summer and Smoke (1948), and two short stories written in parallel, "The Yellow Bird" (1941-1947) and "One Arm" (1942-1948). To a lesser extent, The Glass Menagerie (1945) and A Streetcar Named Desire (1947) will also be discussed. The short stories will allow us to consider that these plays, and perhaps Williams' theater in general, embrace an ambivalent but constructive sense of laughter. Laughter in Williams' plays is almost invisible; still it sets action in motion, haunts the characters and roams like a doppelgänger between words and phrases to illuminate what is dark and demystify what is poignant. In this essay I contend that Williams' plays are not what they appear to be: laughter endows the action with a salutary haunting authority stemming from autobiographic and perhaps traumatic material which eventually drains pathos offering wit instead.

\section{INDEX}

Keywords : Tennessee Williams, Oscar Wilde, poetics, short story, drama, myth, ritual, homosexuality, haunting, image, rewriting, Greece, Iacchos, Baubô, anasyrma

Mots-clés : Tennessee Williams, Oscar Wilde, poétique, nouvelle, théâtre, mythe, rituel, homosexualité, hantise, image, réécriture, Grèce, Iacchos, Baubô, anasyrma

\section{AUTEUR}

EMMANUEL VERNADAKIS

Professeur

CIRPaLL, Université d'Angers

Emmanuel.vernadakis@univ-angers.fr 